\title{
End-to-End Data Rate Performance of Decode-and-Forward Relaying with Different Resource Allocation Schemes
}

\author{
Inam Ullah,, Alexis Dowhuszko, ${ }^{2}$ Zhong Zheng, ${ }^{3}$ \\ David González González, ${ }^{1}$ and Jyri Hämäläinen ${ }^{1}$ \\ ${ }^{1}$ Department of Communications and Networking, Aalto University, Espoo, Finland \\ ${ }^{2}$ Centre Tecnologic Telecomunicacions Catalunya, Barcelona, Spain \\ ${ }^{3}$ Department of Computer Science, University of Texas at Dallas, Richardson, TX, USA \\ Correspondence should be addressed to Inam Ullah; inam.ullah@aalto.fi
}

Received 19 June 2017; Revised 1 September 2017; Accepted 18 September 2017; Published 26 October 2017

Academic Editor: Massimo Condoluci

Copyright (C) 2017 Inam Ullah et al. This is an open access article distributed under the Creative Commons Attribution License, which permits unrestricted use, distribution, and reproduction in any medium, provided the original work is properly cited.

\begin{abstract}
This paper studies the end-to-end (e2e) data rate of dual-hop Decode-and-Forward (DF) infrastructure relaying under different resource allocation schemes. In this context, we first provide a comparative analysis of the optimal resource allocation scheme with respect to several other approaches in order to provide insights into the system behavior and show the benefits of each alternative. Then, assuming the optimal resource allocation, a closed form expression for the distribution of the mean and outage data rates is derived. It turns out that the corresponding mean e2e data rate formula attains an expression in terms of an integral that does not admit a closed form solution. Therefore, a tight lower bound formula for the mean e2e data rate is presented. Results can be used to select the most convenient resource allocation scheme and perform link dimensioning in the network planning phase, showing the explicit relationships that exist between component link bandwidths, SNR values, and mean data rate.
\end{abstract}

\section{Introduction}

The mobile communication systems are constantly evolving due to increasing demand for higher data rates, better coverage, and improved services. For example, there are almost 7.6 billion mobile subscriptions enabled globally by cellular network operators by first quarter (Q1) of 2017. It is observed that currently there are 3.9 billion mobile smartphone subscriptions, 4.6 billion mobile broadband subscriptions, and 2.1 billion Long Term Evolution (LTE) subscriptions which are expected to reach to 6.8 billion, 8.3 billion, and 5 billion, respectively, by year 2022 [1]. Moreover, it is expected that the global mobile data traffic will be 49 exabytes monthly by 2021 [2]. Moreover, the Internet-of-Things (IoT) and Ehealth services are the emerging drivers along with the other growing range of vertical applications and business models [3].

The increasing demand for better data rates and coverage has led to stringent performance requirements for radio access networks. Since relaying is seen as a promising technology to improve the mobile networks coverage and capacity, Relay Nodes (RNs) are expected to form an integral part of the future mobile network infrastructure. Accordingly, relaying has been included in standards of Third-Generation Partnership Project (3GPP) Long-Term Evolution Release 10 and beyond (LTE-Advanced), and it is also expected to play a key role in the 5th-generation (5G) networks.

Relaying technologies can be classified into several groups according to their operation modes. This papers focuses on the so-called infrastructure (fixed) relaying, which assumes that the locations of the RNs are selected by the cellular network provider in the network planning phase to extend the cellular coverage and enhance the capacity of the mobile network within its coverage area [4]. Due to that, the transmission power of infrastructure RNs is usually kept constant to keep under control the harmful cochannel interference towards the adjacent cells. Infrastructure relays can be broadly divided into two groups: Amplify-and-Forward (AF) and Decode-and-Forward (DF). In AF, RNs directly amplify the signal that is received from/to the base station (BS) 


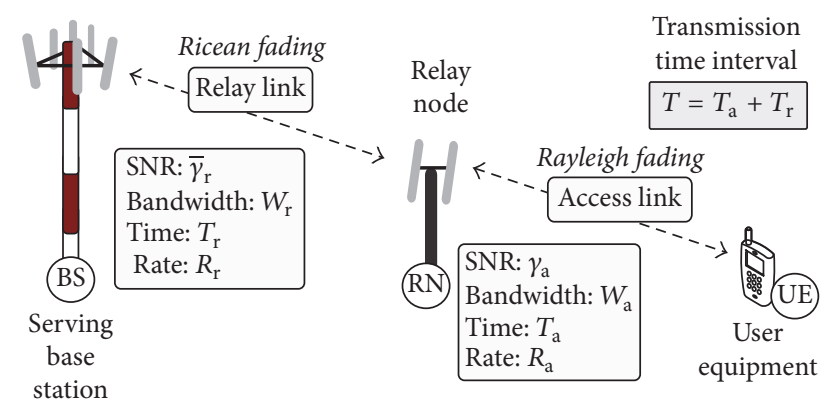

FIGURE 1: System model for the infrastructure relaying system consisting of a BS, RN, and UE. Fast fading component of the wireless channel in the RL and AL is modeled according to a Ricean (LoS) and Rayleigh (NLoS) distribution.

before its retransmission to/from the User Equipment (UE). On the other hand, DF RN decodes the information from the received signal before its retransmission towards the destination. In the following, the focus will be on the end-toend (e2e) performance of the infrastructure DF relaying. By terms relay link (RL) and Access Link (AL), we refer to the BS-RN and RN-UE links, respectively, as shown in Figure 1.

Decode-and-Forward relaying has been extensively studied in the literature. The performance and energy efficiency of relay enhanced networks have gained a lot of interest; see, for example, [5-15]. Recently the impact of buffer on the performance of DF relaying has also been investigated in references like [16]. Yet, especially in analytical studies, the performance analysis of DF relaying systems has been conducted under the assumption that the time-frequency resources are equally shared in long-term between both RL and AL links [17-22]. In some recent works $[23,24]$, the notion of optimal resource allocation (RA) introduced in [25] has also been considered. We recall that resource allocation is said to be optimal when the same amount of data is transferred over both RL and AL at each radio frame. A more formal definition of this concept is presented in Section 3. In this context, different authors have studied the relaying performance in terms of enhanced spectral efficiency by proposing the implementation of rate adaptation and relay selection scheme [23], as well as adaptive modulation schemes [24]. Nevertheless, to the best of our knowledge, the analysis of the e2e data rate and the derivation of closed form expressions for its distribution in presence of optimal resource allocation have not been addressed in the literature before.

Contributions. The main contributions of this paper are threefold. Firstly, we carry out a comparative analysis of several resource allocation schemes; that is, conventional RA (where the RL and AL equally share the time resources), fixed RA (where the RL and AL resources are proportionally allocated according to their corresponding mean data rates), and optimal RA (where the RL and AL resources are allocated according to the instantaneous data rates in each link). Secondly, assuming the optimal RA scheme is used, we derive a closed form expression for the probability distribution function for the mean and outage data rate of the two-hop DF relaying system. Finally, when using the optimal RA scheme, the mean e2e data rate attains an expression in terms of an integral that does not admit closed form solution. Therefore we derive a tight lower bound formula that approximates the mean e2e data rate performance successfully.

The rest of the paper is organized as follows: Section 2 presents the system model and the corresponding channel modeling assumptions. Section 3 explains the different RA schemes to be studied, characterizing the differences between conventional RA, fixed RA with/without buffering, and optimal RA. Closed form expressions that characterize the instantaneous, mean, and outage data rates of the DF relaying system with optimal RA are presented in Section 4. Conclusions are drawn in Section 5, whereas future extensions of the research done in this paper are briefly discussed in Section 6 .

\section{System Model}

The two-hop relay system that is considered in this paper is shown in Figure 1. In this model, the communication between UE and BS always takes place using the RN as an intermediate node. A radio frame of duration $T$ is assumed, where communication resources are time-shared between both AL and RL assuming an infinitesimal granularity. Halfduplex operation is considered, that is, $\mathrm{RN}$ cannot transmit and receive information simultaneously; hence, $T=T_{\mathrm{r}}+T_{\mathrm{a}}$, where $T_{\mathrm{r}}$ and $T_{\mathrm{a}}$ correspond to the time allocated to the RL and AL transmission, respectively.

Hereafter, the subscripts "r" and "a" are used to refer to the $\mathrm{RL}$ and AL, respectively. The achievable data rate that each link is able to support depends on its allocated bandwidth and received SNR. Thus, according to Shannon's formula, the achievable rate during the Transmission Time Interval (TTI) that corresponds to each radio frame is given by

$$
\begin{aligned}
& R_{\mathrm{r}}=W_{\mathrm{r}} \log _{2}\left(1+\gamma_{\mathrm{r}}\right), \\
& R_{\mathrm{a}}=W_{\mathrm{a}} \log _{2}\left(1+\gamma_{\mathrm{a}}\right),
\end{aligned}
$$

where $W_{\mathrm{r}}$ and $W_{\mathrm{a}}$ correspond to the bandwidth allocated to each link, and $\gamma_{\mathrm{r}}$ and $\gamma_{\mathrm{a}}$ represent instantaneous SNR values, which are assumed to be constant during each TTI but may vary between TTIs according to fast fading statistics. In infrastructure relaying, RNs form a fixed part of the network and their locations are carefully planned during the deployment phase, such that a strong Line-of-Sight (LoS) component exists towards the serving BS with high enough probability. Indeed, channel models for practical systems do consider scenarios where LoS component exists in the relay link, for example, [26]. In these communication scenarios, low variability in the SNR of the RL is expected and, hence, a Ricean distribution can be used to model the fast fading component of the wireless channel in the RL [27, 28]. In the $\mathrm{AL}$, on the other hand, a Non-Line-of-Sight (NLoS) condition is considered as UE location is typically random and variable; hence, a Rayleigh distribution can be used to model the fast fading component of the wireless channel in the $\mathrm{AL}$, as shown in Figure 1. 


\section{Resource Allocation}

This section presents the different RA schemes that can be used according to the flexibility that the two-hop DF relaying system has to update the fraction of communication resources that are allocated to both RL and AL. Four different RA schemes are analyzed: conventional RA, fixed RA with/without buffer, and optimal RA.

3.1. Conventional $R A$. In this scheme $[17,29]$, the time is equally divided between RL and AL, that is, $T_{\mathrm{a}}=T_{\mathrm{r}}=0.5 \cdot \mathrm{T}$. Thus, the conventional e2e data rate $\left(R_{\mathrm{e} 2 \mathrm{e}}^{\text {conv }}\right)$ is given by

$$
R_{\mathrm{e} 2 \mathrm{e}}^{\mathrm{conv}}=\frac{1}{2} \min \left\{R_{\mathrm{r}}, R_{\mathrm{a}}\right\} .
$$

In other words, the performance of both links depends upon their corresponding SNRs, and the link experiencing the lowest SNR will represent the bottleneck for the overall e2e data rate of the relaying system. Here, the factor $1 / 2$ is due to the half-duplex constraint.

3.2. Fixed RA without Buffer. In this type of RA scheme, the values of $T_{\mathrm{a}}$ and $T_{\mathrm{r}}$ are selected proportionally to the mean data rate of each link, that is, $\mathbb{E}\left\{R_{\mathrm{r}}\right\}$ and $\mathbb{E}\left\{R_{\mathrm{a}}\right\}$. Hence, we can write the rate without buffer (denoted by $R_{\mathrm{e} 2 \mathrm{e}}^{\mathrm{wob}}$ ) as

$$
T R_{\mathrm{e} 2 \mathrm{e}}^{\mathrm{wob}}=T_{\mathrm{a}} \mathbb{E}\left\{R_{\mathrm{a}}\right\}=T_{\mathrm{r}} \mathbb{E}\left\{R_{\mathrm{r}}\right\} .
$$

From (3), we obtain

$$
\begin{aligned}
& T_{\mathrm{a}}=\frac{T_{\mathrm{r}} \mathbb{E}\left\{R_{\mathrm{r}}\right\}}{\mathbb{E}\left\{R_{\mathrm{a}}\right\}}, \\
& T_{\mathrm{r}}=\frac{T_{\mathrm{a}} \mathbb{E}\left\{R_{\mathrm{a}}\right\}}{\mathbb{E}\left\{R_{\mathrm{r}}\right\}} .
\end{aligned}
$$

Since in general $\mathbb{E}\left\{R_{\mathrm{r}}\right\} \neq \mathbb{E}\left\{R_{\mathrm{a}}\right\}$, the time allocations for each link will be different, that is, $T_{\mathrm{r}} \neq T_{\mathrm{a}}$, verifying $T_{\mathrm{a}}+T_{\mathrm{r}}=$ $T$. Hence, the overall payload data transmitted over the e2e link is

$$
P=\min \left\{T_{\mathrm{a}} R_{\mathrm{a}}, T_{\mathrm{r}} R_{\mathrm{r}}\right\} .
$$

Using (5), the e2e data rate can be written as follows:

$$
\begin{aligned}
& R_{\mathrm{e} 2 \mathrm{e}}^{\text {wob }}=\frac{P}{T}=\frac{\min \left\{T_{\mathrm{a}} R_{\mathrm{a}}, T_{\mathrm{r}} R_{\mathrm{r}}\right\}}{T}, \\
& \quad=\frac{1}{T} \min \left\{\frac{T_{\mathrm{r}} \mathbb{E}\left\{R_{\mathrm{r}}\right\}}{\mathbb{E}\left\{R_{\mathrm{a}}\right\}} R_{\mathrm{a}}, T_{\mathrm{r}} R_{\mathrm{r}}\right\}, \\
& =\frac{1}{T_{\mathrm{r}}+\left(T_{\mathrm{r}} \mathbb{E}\left\{R_{\mathrm{r}}\right\} / \mathbb{E}\left\{R_{\mathrm{a}}\right\}\right)} \min \left\{\frac{T_{\mathrm{r}} \mathbb{E}\left\{R_{\mathrm{r}}\right\}}{\mathbb{E}\left\{R_{\mathrm{a}}\right\}} R_{\mathrm{a}}, T_{\mathrm{r}} R_{\mathrm{r}}\right\},
\end{aligned}
$$

where the second equality follows from (4) and the third equality holds since $T=T_{\mathrm{r}}+T_{\mathrm{a}}=T_{\mathrm{r}}+T_{\mathrm{r}} \cdot \mathbb{E}\left\{R_{\mathrm{r}}\right\} / \mathbb{E}\left\{R_{\mathrm{a}}\right\}$.
Furthermore, we can apply the formula $\min \{X, Y\}=1 / 2(X+$ $Y-|Y-X|)$ in (6) and obtain

$$
\begin{aligned}
R_{\mathrm{e} 2 \mathrm{e}}^{\mathrm{wob}}= & \frac{1}{2} \frac{\mathbb{E}\left\{R_{\mathrm{a}}\right\}}{\mathbb{E}\left\{R_{\mathrm{a}}\right\}+\mathbb{E}\left\{R_{\mathrm{r}}\right\}} \\
& \times\left[\frac{\mathbb{E}\left\{R_{\mathrm{r}}\right\}}{\mathbb{E}\left\{R_{\mathrm{a}}\right\}} R_{\mathrm{a}}+R_{\mathrm{r}}-\left|\frac{\mathbb{E}\left\{R_{\mathrm{r}}\right\}}{\mathbb{E}\left\{R_{\mathrm{a}}\right\}} R_{\mathrm{a}}-R_{\mathrm{r}}\right|\right] .
\end{aligned}
$$

Three different closed form e2e data rate solutions can be derived in this situation solving the modulus operator; that is,

$$
R_{\mathrm{e} 2 \mathrm{e}}^{\text {wob }}= \begin{cases}\frac{R_{\mathrm{r}} \mathbb{E}\left\{R_{\mathrm{a}}\right\}}{\mathbb{E}\left\{R_{\mathrm{r}}\right\}+\mathbb{E}\left\{R_{\mathrm{a}}\right\}}, & \frac{\mathbb{E}\left\{R_{\mathrm{r}}\right\}}{\mathbb{E}\left\{R_{\mathrm{a}}\right\}} R_{\mathrm{a}}>R_{\mathrm{r}}, \\ \frac{R_{\mathrm{a}} \mathbb{E}\left\{R_{\mathrm{r}}\right\}}{\mathbb{E}\left\{R_{\mathrm{r}}\right\}+\mathbb{E}\left\{R_{\mathrm{a}}\right\}}, & \frac{\mathbb{E}\left\{R_{\mathrm{r}}\right\}}{\mathbb{E}\left\{R_{\mathrm{a}}\right\}} R_{\mathrm{a}}<R_{\mathrm{r}}, \\ \frac{1}{2} \frac{R_{\mathrm{r}} \mathbb{E}\left\{R_{\mathrm{a}}\right\}+R_{\mathrm{a}} \mathbb{E}\left\{R_{\mathrm{r}}\right\}}{\mathbb{E}\left\{R_{\mathrm{r}}\right\}+\mathbb{E}\left\{R_{\mathrm{a}}\right\}}, & \frac{\mathbb{E}\left\{R_{\mathrm{r}}\right\}}{\mathbb{E}\left\{R_{\mathrm{a}}\right\}} R_{\mathrm{a}}=R_{\mathrm{r}} .\end{cases}
$$

3.3. Fixed RA with Buffer. In this RA scheme, it is assumed that RNs have large buffers to prevent data overflows during transmission. In buffer-aided relaying, RN can better use the Channel State Information while selecting between reception in RL and transmission in AL. Buffer also makes it possible for $\mathrm{RN}$ to effectively utilize the temporal diversity in scheduling. In this relaying type, the $\mathrm{BS}$ can continue the transmission on the RL regardless of the channel quality in the AL. In case of deep fading in the AL, data can be stored at the RN's buffer until the AL channel condition becomes suitable for transmission again. Once, the AL channel condition is favorable, then, the RN can forward the previously buffered data to the UE [30-32]. In this setting, we denote the e2e data rate by $R_{\mathrm{e} 2 \mathrm{e}}^{\mathrm{wb}}$. Since the same amount of data is transferred over both $\mathrm{RL}$ and $\mathrm{AL}$ in long-term, the relation

$$
T \cdot R_{\mathrm{e} 2 \mathrm{e}}^{\mathrm{wb}}=T_{\mathrm{a}} \cdot \mathbb{E}\left\{R_{\mathrm{a}}\right\}=T_{\mathrm{r}} \cdot \mathbb{E}\left\{R_{\mathrm{r}}\right\}
$$

should hold. Then, we find that

$$
\begin{aligned}
\mathbb{E}\left\{R_{\mathrm{e} 2 \mathrm{e}}^{\mathrm{wb}}\right\} & =\frac{T_{\mathrm{r}} \cdot \mathbb{E}\left\{R_{\mathrm{r}}\right\}}{T_{\mathrm{r}}+T_{\mathrm{a}}}=\frac{\mathbb{E}\left\{R_{\mathrm{r}}\right\}}{1+T_{\mathrm{a}} / T_{\mathrm{r}}} \\
& =\frac{\mathbb{E}\left\{R_{\mathrm{r}}\right\} \mathbb{E}\left\{R_{\mathrm{a}}\right\}}{\mathbb{E}\left\{R_{\mathrm{r}}\right\}+\mathbb{E}\left\{R_{\mathrm{a}}\right\}} .
\end{aligned}
$$

3.4. Optimal RA. The notion of optimal resource sharing for $N$-hops connections was developed in [25]. In this scheme, the e2e data rate $\left(R_{\mathrm{e} 2 \mathrm{e}}^{\mathrm{opt}}\right)$ is maximized when the same amount of data is transferred over each link at each TTI. This is equivalent to verify that

$$
T R_{\mathrm{e} 2 \mathrm{e}}^{\mathrm{opt}}=T_{\mathrm{r}} R_{\mathrm{r}}=T_{\mathrm{a}} R_{\mathrm{a}}
$$

holds for each radio frame. In order to achieve condition (11), proper resource allocation is required; that is, the selection of $T_{\mathrm{r}}, T_{\mathrm{a}}, W_{\mathrm{r}}$, and $W_{\mathrm{a}}$ depends on the instantaneous SNR of 
each link (in principle, adjusting power is another degree of freedom; however, this work deals with infrastructure fixed relays, where the transmission power of BSs and RNs in downlink is kept constant to avoid potentially harmful cochannel interference towards the adjacent cells). Hence, time and bandwidth are the degrees of freedom to achieve (11). Practical implementations, such as Type 2 DF relaying in LTE-A and Mobile WiMAX [33], provide means to conveniently allocate time-frequency resources, such that optimal resourcing can be achieved or at least well approximated (due to the existence of a practical granularity in the partition of resources). Thus, using (1) and (11), the expression for the optimal e2e data rate is as follows:

$$
R_{\mathrm{e} 2 \mathrm{e}}^{\mathrm{opt}}=\frac{T_{\mathrm{r}} R_{\mathrm{r}}}{T_{\mathrm{r}}+T_{\mathrm{a}}}=\frac{R_{\mathrm{r}}}{1+T_{\mathrm{a}} / T_{\mathrm{r}}}=\frac{R_{\mathrm{r}}}{1+R_{\mathrm{r}} / R_{\mathrm{a}}}=\frac{R_{\mathrm{r}} R_{\mathrm{a}}}{R_{\mathrm{r}}+R_{\mathrm{a}}} .
$$

3.5. Performance Comparison of the Different RA Schemes. When we compare the efficiency of the different RA schemes that have been presented so far, it is possible to show that

$$
\mathbb{E}\left\{R_{\mathrm{e} 2 \mathrm{e}}^{\mathrm{wb}}\right\} \geq \mathbb{E}\left\{R_{\mathrm{e} 2 \mathrm{e}}^{\mathrm{opt}}\right\} \geq \mathbb{E}\left\{R_{\mathrm{e} 2 \mathrm{e}}^{\mathrm{wob}}\right\} \geq \mathbb{E}\left\{R_{\mathrm{e} 2 \mathrm{e}}^{\mathrm{conv}}\right\}
$$

holds. Since the conventional RA scheme does not have a buffer at the $\mathrm{RN}$ and always divides the communications resources equally between both $\mathrm{RL}$ and $\mathrm{AL}$, its performance is clearly inferior to the one that corresponds to the other relaying strategies. Yet, conventional RA is the simplest scheme to allocate resources in practice. The fixed RA without buffer adjusts the transmission times of the radio frames to fit with the long-term (expected) data rates that each individual link is able to support; see (3). However, due to the absence of a buffer at the $\mathrm{RN}$, its e2e data rate is inferior not only to the one that corresponds to the fixed RA scheme with buffer, but also to the optimal RA scheme that adjusts the transmission times and rates in RL and AL without delay; see (11). Finally, we note that fixed RA with buffer also beats the optimal RA strategy given in (12) when expected e2e data rates are compared. While there can be $R_{\mathrm{e} 2 \mathrm{e}}^{\mathrm{opt}}>R_{\mathrm{e} 2 \mathrm{e}}^{\mathrm{wb}}$ for instantaneous data rates, in fixed RA with buffer it is possible to support $R_{\mathrm{r}}=\mathbb{E}\left\{R_{\mathrm{r}}\right\}$ and $R_{\mathrm{a}}=\mathbb{E}\left\{R_{\mathrm{a}}\right\}$. Note also that Jensen's inequality verifies $\mathbb{E}\left\{R_{\mathrm{e} 2 \mathrm{w}}^{\mathrm{wb}}\right\} \geq \mathbb{E}\left\{R_{\mathrm{e} 2 \mathrm{e}}^{\mathrm{opt}}\right\}$; see (12) and (13); nevertheless, it is worth noting that the use of buffers introduces additional latency (and additional hardware costs), as the data blocks transported over the $\mathrm{RL}$ and $\mathrm{AL}$ of a radio frame do not usually match over the same TTI.

Figure 2 presents the Cumulative Distribution Function (CDF) of the gains that can be achieved by means of the optimal RA with respect to the aforementioned baseline schemes (i.e., conventional RA and fixed RA with and without buffer). Optimal RA always provides significant gains with respect to conventional and fixed RA without using buffer. We recall that in case of the fixed RA with buffers it is possible to support the case with $R_{\mathrm{r}}=\mathbb{E}\left\{R_{\mathrm{r}}\right\}$ and $R_{\mathrm{a}}=\mathbb{E}\left\{R_{\mathrm{a}}\right\}$; hence, sometimes $R_{\mathrm{e} 2 \mathrm{e}}^{\mathrm{opt}}<R_{\mathrm{e} 2 \mathrm{e}}^{\mathrm{wb}}$, as shown in Figure 2. Yet, $R_{\mathrm{e} 2 \mathrm{e}}^{\mathrm{opt}}$ represent the rate-optimal RA strategy provided that a buffer is not available at the RN.

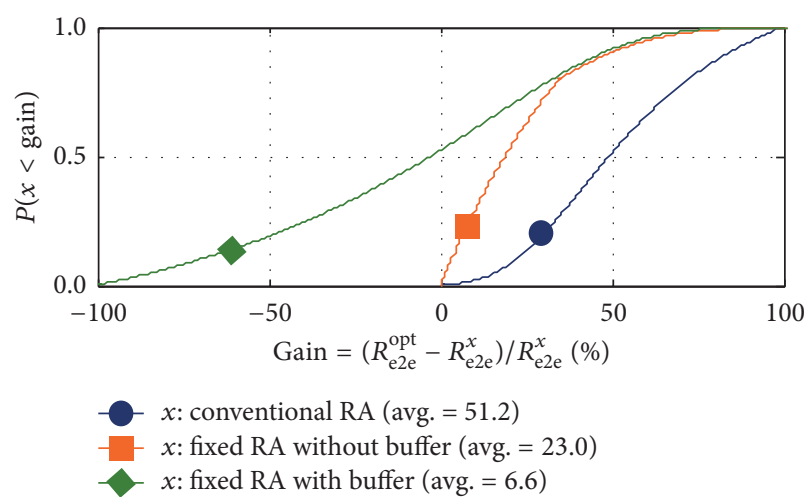

FIGURE 2: Cumulative Distribution Function of the instantaneous gains that optimal RA provides with respect to the other baseline RA schemes in a two-hop DF relaying (at TTI level). Parameters: $W_{\mathrm{r}}=W_{\mathrm{a}}=180 \mathrm{kHz}, \mathbb{E}\left\{\gamma_{\mathrm{r}}\right\}=\bar{\gamma}_{\mathrm{r}}=15 \mathrm{~dB}($ Ricean $K$-factor $=12 \mathrm{~dB})$, and $\mathbb{E}\left\{\gamma_{\mathrm{a}}\right\}=\bar{\gamma}_{\mathrm{a}}=5 \mathrm{~dB}$. Ideal Channel State Information is assumed in transmission.

3.6. Role of the Channel State Information. To implement a RA scheme both long- and short-term Channel State Information (CSI) can be used. To keep the discussion simple, we focus on the downlink direction of communication and assume that receiver has always short-term CSI (obtained from pilot signals through a suitable channel estimation process), while transmitter may have either long- or shortterm CSI only if the receiver provides it through a certain feedback mechanism (i.e., Frequency Division Duplex air interface). Feedback is fast for short-term CSI and slow for long-term CSI. Here, "fast" and "slow" refer to the speed of the feedback provision when compared to the coherence time of the wireless channel.

We note that any RA scheme that relies on instantaneous rates (i.e., $R_{\mathrm{r}}$ and $R_{\mathrm{a}}$ ) should have short-term CSI of both RL and AL to make the RA decisions in each TTI. This is the case for the optimal RA scheme, where RN should request CSI report from the UE before performing the allocation of resources (jointly with BS) over both RL and AL. In fixed RA without buffer, transmission times $T_{\mathrm{r}}$ and $T_{\mathrm{a}}$ are selected proportionally to the mean data rate of each link. Therefore, only long-term CSI is needed to select the allocation of resources which, after being determined, is kept fixed for all TTI. Similarly, fixed RA with buffer applies only long-term CSI. Finally, if fast radio layer scheduling is applied in a radio link, then short-term CSI is always needed.

\section{End-to-End Data Rate for Optimal RA}

In this section we focus on characterizing the data performance of the optimal RA scheme, which adjusts the time sharing fraction of communication resources allocated to both RL and AL on a TTI basis. The CDF of the e2e data rate is first characterized and, after that, closed form expressions for both the mean and the outage data rates are also obtained. 


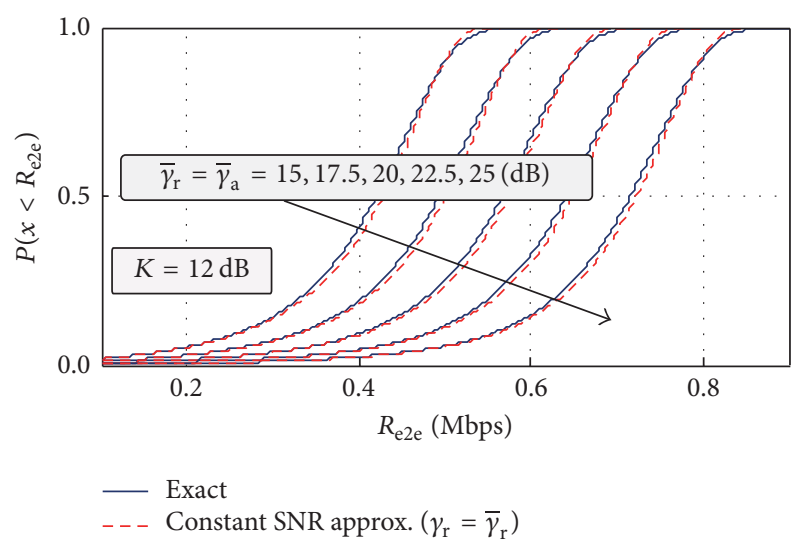

FIGURE 3: Accuracy of the constant SNR assumption for the channel quality distribution in the RL. The CDF of the $R_{\mathrm{e} 2 \mathrm{e}}$ is presented here for different combinations of mean SNR in both $\operatorname{RL}\left(\bar{\gamma}_{\mathrm{r}}\right)$ and $\operatorname{AL}\left(\bar{\gamma}_{\mathrm{a}}\right)$.

4.1. Approximation of the SNR Distribution in the Relay Link. By combining (1) and (12), the e2e data rate of optimal RA scheme can be expressed as follows:

$$
R_{\mathrm{e} 2 \mathrm{e}}^{\mathrm{opt}}=\frac{W_{\mathrm{r}} \log _{2}\left(1+\gamma_{\mathrm{r}}\right) W_{\mathrm{a}} \log _{2}\left(1+\gamma_{\mathrm{a}}\right)}{W_{\mathrm{r}} \log _{2}\left(1+\gamma_{\mathrm{r}}\right)+W_{\mathrm{a}} \log _{2}\left(1+\gamma_{\mathrm{a}}\right)},
$$

where $\gamma_{\mathrm{r}}$ and $\gamma_{\mathrm{a}}$ are the instantaneous SNRs of the RL and $\mathrm{AL}$, respectively. If the fast fading components of these wireless links admit Ricean (LoS) and Rayleigh (NLoS) fading distributions, then the Probability Density Functions (PDFs) for $\gamma_{\mathrm{r}}$ and $\gamma_{\mathrm{a}}$ are given by [28]

$$
\begin{aligned}
& f_{\gamma_{\mathrm{r}}}(\gamma) \\
& \quad=\frac{K+1}{\bar{\gamma}_{\mathrm{r}}} e^{-\left(K \bar{\gamma}_{\mathrm{r}}+(K+1) \gamma\right) / \bar{\gamma}_{\mathrm{r}}} I_{0}\left(2 \sqrt{\frac{K(K+1) \gamma}{\bar{\gamma}_{\mathrm{r}}}}\right), \\
& f_{\gamma_{\mathrm{a}}}(\gamma)=\frac{1}{\bar{\gamma}_{\mathrm{a}}} e^{-\gamma / \bar{\gamma}_{\mathrm{a}}},
\end{aligned}
$$

respectively. In these equations, $\bar{\gamma}_{\mathrm{r}}$ and $\bar{\gamma}_{\mathrm{a}}$ represent the mean SNR of the RL and AL, respectively. Furthermore, $I_{0}$ is the zero-order modified Bessel function of the first kind [34], which makes the mathematical analysis of (14) challenging. However, in practical situations with strong LoS conditions, the Ricean $K$-factor can be large; see, for example, the analysis provided in [27], where typical value in the order of $12 \mathrm{~dB}$ can be easily observed. Presuming a high $K$-factor, we assume in the analysis that the SNR is constant in the LoS link between BS and RN. Although results of [27] provide a good justification for this assumption, we verify it through simulations in the following. Figure 3 shows the CDF of $R_{\mathrm{e} 2 \mathrm{e}}^{\mathrm{opt}}$ in (14), considering that the SNR in the AL is always distributed according to (16). For the SNR in the RL, we consider two cases: Firstly, we assume that fast fading in the RL is distributed according to (15); then, we consider a constant SNR for the RL $\left(\gamma_{\mathrm{r}}=\bar{\gamma}_{\mathrm{r}}\right)$. Figure 3 shows the CDF of $R_{\mathrm{e} 2 \mathrm{e}}^{\mathrm{opt}}$ for different $\bar{\gamma}_{\mathrm{r}}$ and $\bar{\gamma}_{\mathrm{a}}$, with $K=12 \mathrm{~dB}$ in all cases. Results show that the constant SNR assumption is valid not only in terms of the resulting distributions, but also in terms of the deviation of the average $R_{\mathrm{e} 2 \mathrm{e}}^{\mathrm{opt}}$, where for values of $K>12 \mathrm{~dB}$ it was verified that the approximation error is always smaller than $1 \%$ for the whole data rate range under consideration. Indeed, assuming a constant rate for the RL (i.e., $R_{\mathrm{r}}$ constant), this behavior is also justified by Jensen's inequality as follows:

$$
\mathbb{E}\left\{R_{\mathrm{r}}\right\}=\mathbb{E}\left\{\log _{2}\left(1+\gamma_{\mathrm{r}}\right)\right\} \leq \log _{2}\left(1+\mathbb{E}\left\{\gamma_{\mathrm{r}}\right\}\right),
$$

where the lower bound for the mean e2e data rate is asymptotically tight as the $K$-factor of the Ricean distribution grows.

4.2. Probability Distribution for the Instantaneous e2e Data Rate. Starting from (12), the CDF $F_{R_{\mathrm{e} 2 \mathrm{e}}^{\text {opt }}}$ can be obtained by a standard change of a random variable [35]. First, by setting

$$
r_{\mathrm{e} 2 \mathrm{e}}=g_{1}\left(R_{\mathrm{a}}\right)=\frac{R_{\mathrm{r}} R_{\mathrm{a}}}{\left(R_{\mathrm{r}}+R_{\mathrm{a}}\right)}
$$

and solving $R_{\mathrm{a}}$ in terms of $r_{\mathrm{e} 2 \mathrm{e}}$, it is possible to write

$$
R_{\mathrm{a}}=g_{1}^{-1}\left(r_{\mathrm{e} 2 \mathrm{e}}\right)=\frac{\left(r_{\mathrm{e} 2 \mathrm{e}} R_{\mathrm{r}}\right)}{\left(R_{\mathrm{r}}-r_{\mathrm{e} 2 \mathrm{e}}\right)} .
$$

The CDF of $R_{\mathrm{e} 2 \mathrm{e}}^{\mathrm{opt}}$ admits the following formulation:

$$
F_{R_{\mathrm{e} 2 \mathrm{e}}^{\text {opt }}}\left(r_{\mathrm{e} 2 \mathrm{e}}\right)=F_{R_{\mathrm{a}}}\left(g_{1}^{-1}\left(r_{\mathrm{e} 2 \mathrm{e}}\right)\right)=F_{R_{\mathrm{a}}}\left(\frac{r_{\mathrm{e} 2 \mathrm{e}} R_{\mathrm{r}}}{R_{\mathrm{r}}-r_{\mathrm{e} 2 \mathrm{e}}}\right),
$$

where $r_{\mathrm{e} 2 \mathrm{e}}<R_{\mathrm{r}}$. Second, the SNR in the AL can be expressed as a function of $r_{\mathrm{a}}$ as follows:

$$
\gamma_{\mathrm{a}}=g_{2}^{-1}\left(r_{\mathrm{a}}\right)=\exp \left(\frac{\ln 2}{W_{\mathrm{a}}} r_{\mathrm{a}}\right)-1 .
$$

Then, it is possible to write

$$
F_{R_{\mathrm{a}}}\left(r_{\mathrm{a}}\right)=F_{\gamma_{\mathrm{a}}}\left(g_{2}^{-1}\left(r_{\mathrm{a}}\right)\right)=F_{\gamma_{\mathrm{a}}}\left(e^{\left(\ln 2 / W_{\mathrm{a}}\right) r_{\mathrm{a}}}-1\right) .
$$

By combining (12), (20), and (22), $F_{R_{\mathrm{e} 2 \mathrm{e}}^{\mathrm{opt}}}$ is given by

$$
F_{R_{\mathrm{e} 2 \mathrm{e}}^{\mathrm{opt}}}\left(r_{\mathrm{e} 2 \mathrm{e}}\right)=F_{\gamma_{\mathrm{a}}}\left(e^{\left(\ln 2 / W_{\mathrm{a}}\right)\left(r_{\mathrm{e} 2 \mathrm{e}} R_{\mathrm{r}} /\left(R_{\mathrm{r}}-r_{\mathrm{e} 2 \mathrm{e}}\right)\right)}-1\right),
$$

where $F_{R_{\mathrm{e} 2 \mathrm{e}}^{\mathrm{opt}}}\left(r_{\mathrm{e} 2 \mathrm{e}}\right)=0$ if $r_{\mathrm{e} 2 \mathrm{e}}<0$ and $F_{R_{\mathrm{e} 2 \mathrm{e}}^{\mathrm{opt}}}\left(r_{\mathrm{e} 2 \mathrm{e}}\right)=1$ if $r_{\mathrm{e} 2 \mathrm{e}} \geq R_{\mathrm{r}}$. Thus, using $(16), F_{R_{\text {e2e }}^{\text {opt }}}$ becomes

$$
F_{R_{\mathrm{e} 2 \mathrm{e}}^{\mathrm{opt}}}\left(r_{\mathrm{e} 2 \mathrm{e}}\right)=1-e^{-\left(1 / \bar{\gamma}_{\mathrm{a}}\right)\left(e^{\left(\ln 2 / W_{\mathrm{a}}\right)\left(r_{\mathrm{e} 2 \mathrm{e}} R_{\mathrm{r}} /\left(R_{\mathrm{r}}-r_{\mathrm{e} 2 \mathrm{e}}\right)\right)}-1\right)} .
$$

Using (23), the PDF for $R_{\mathrm{e} 2 \mathrm{e}}^{\mathrm{opt}}$ can also be deduced in cases where $\gamma_{\mathrm{a}}$ has another distribution, such as Nakagami or Weibull [28]. By taking the derivative of (24), the PDF of $R_{\mathrm{e} 2 \mathrm{e}}^{\mathrm{opt}}$ is given by

$$
\begin{aligned}
& f_{R_{\mathrm{e} 2 \mathrm{e}}^{\mathrm{opt}}}\left(r_{\mathrm{e} 2 \mathrm{e}}\right) \\
& =\frac{\ln 2}{\bar{\gamma}_{\mathrm{a}} W_{\mathrm{a}}} \exp \left(-\frac{\exp \left(\ln 2 R_{\mathrm{r}} r_{\mathrm{e} 2 \mathrm{e}} / W_{\mathrm{a}}\left(R_{\mathrm{r}}-r_{\mathrm{e} 2 \mathrm{e}}\right)\right)-1}{\bar{\gamma}_{\mathrm{a}}}\right) \\
& \quad \times \frac{R_{\mathrm{r}}^{2}}{\left(R_{\mathrm{r}}-r_{\mathrm{e} 2 \mathrm{e}}\right)^{2}} \exp \left(\frac{\ln 2 R_{\mathrm{r}} r_{\mathrm{e} 2 \mathrm{e}}}{W_{\mathrm{a}}\left(R_{\mathrm{r}}-r_{\mathrm{e} 2 \mathrm{e}}\right)}\right) .
\end{aligned}
$$




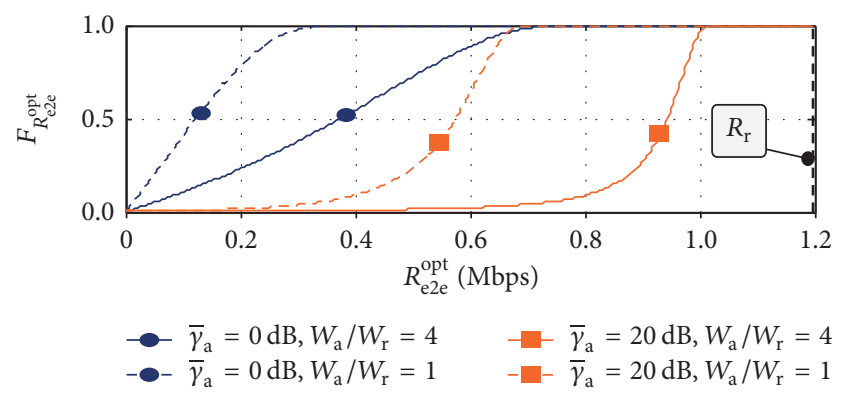

Figure 4: CDF of $R_{\mathrm{e} 2 \mathrm{e}}^{\mathrm{opt}} ; \bar{\gamma}_{\mathrm{a}} \in\{0,20\} \mathrm{dB}, W_{\mathrm{r}}=180 \mathrm{kHz}$, and $\bar{\gamma}_{\mathrm{r}}=$ $20 \mathrm{~dB}$.

The CDF $F_{R_{\text {eze }}^{\text {opt }}}$ is presented in Figure 4, where we have assumed that mean SNR on the RL $\left(\bar{\gamma}_{\mathrm{r}}\right)$ is $20 \mathrm{~dB}$. The dashed vertical line on the right-hand side of the figure depicts the upper limit for the e2e data rate that is obtained if the SNR in the AL grows very large (i.e., upper bound for $R_{\mathrm{e} 2 \mathrm{e}}^{\mathrm{opt}}$. We have also plotted the CDF curves when AL SNR is $0 \mathrm{~dB}$ (dark blue curves) and $20 \mathrm{~dB}$ (orange curves). The impact of bandwidth has been illustrated by setting $W_{\mathrm{a}} / W_{\mathrm{r}}=1$ (dashed curves) and $W_{\mathrm{a}} / W_{\mathrm{r}}=4$ (solid curves).

Results indicate that the imbalance between the mean SNR of the RL and AL can seriously degrade the e2e data rate performance of the optimal RA scheme. If the mean SNR in the AL is notably lower than the mean SNR in the RL (see dark blue curves), then the imbalance can be to some extent compensated by allocating more frequency resources to the AL. Yet, the difference between solid and dashed dark blue curves is largest at the high probability region, while it reduces notably at the low probability region. Thus, if the mean SNR in AL is low, then the use of additional frequency resources in the AL is not necessarily useful to increase the e2e data rate performance.

It is also possible to observe that if the mean SNR in the $\mathrm{RL}$ and $\mathrm{AL}$ is the same (orange curves), then the e2e data rate increases similarly in both low and high probability regions when frequency resources are increased in the AL. Thus, it can be concluded that increasing the frequency resources in the AL is a more effective option when the mean SNR imbalance condition between AL and RL is not notable. We also note that even when AL applies four times larger frequency bandwidth than RL, then the e2e data rate is not very close to the upper bound performance limit. While the e2e data rate of the optimal RA scheme asymptotically approaches the upper limit when $W_{\mathrm{a}} / W_{\mathrm{r}}$ increases, the improved e2e data rate efficiency that is obtained from additional frequency resources in the AL decreases when $W_{\mathrm{a}} / W_{\mathrm{r}}$ becomes larger.

4.3. Mean e2e Data Rate for Optimal RA Scheme. The mean (average) e2e data rate $\left(C_{\text {av }}\right)$ can be obtained by computing the expected value of $R_{\mathrm{e} 2 \mathrm{e}}^{\mathrm{opt}}$ as follows:

$$
C_{\mathrm{av}} \triangleq \mathbb{E}\left\{R_{\mathrm{e} 2 \mathrm{e}}^{\mathrm{opt}}\right\}=\int_{0}^{\infty} r f_{R_{\mathrm{e} 2 \mathrm{e}}}(r) d r .
$$

Assuming constant $\gamma_{\mathrm{r}}$, we proceed by writing (26) in the form

$$
C_{\mathrm{av}}=\int_{0}^{\infty} \frac{R_{\mathrm{r}} W_{\mathrm{a}} \log _{2}\left(1+\gamma_{\mathrm{a}}\right)}{R_{\mathrm{r}}+W_{\mathrm{a}} \log _{2}\left(1+\gamma_{\mathrm{a}}\right)} f_{\gamma_{\mathrm{a}}}\left(\gamma_{\mathrm{a}}\right) d \gamma_{\mathrm{a}}
$$

Thus, $C_{\mathrm{av}}$ is computed for fixed bandwidth in the $\mathrm{AL}\left(W_{\mathrm{a}}\right)$ and instantaneous data rate in the $\mathrm{RL}\left(R_{\mathrm{r}}\right)$. Since $\tau_{\mathrm{r}}=R_{\mathrm{e} 2 \mathrm{e}}^{\mathrm{opt}} / R_{\mathrm{r}}$, the expected time sharing for the RL is given by $\mathbb{E}\left\{\tau_{\mathrm{r}}\right\}=C_{\mathrm{av}} / R_{\mathrm{r}}$. This value can be used if time sharing between RL and AL needs to be fixed. Since $F_{R_{\text {e2e }}^{\text {opt }}}$ is easier to handle than $f_{R_{\text {epe }}^{\text {opt }}}$, integration by parts is used to obtain

$$
C_{\mathrm{av}}=\int_{0}^{\infty}\left(1-F_{R_{\mathrm{e} 2 \mathrm{e}}^{\mathrm{opt}}}(r)\right) d r .
$$

Then, by using (24), substitutions

$$
\begin{gathered}
r=\frac{R_{\mathrm{r}}\left(W_{\mathrm{a}} / \ln 2\right) \ln t}{R_{\mathrm{r}}+\left(W_{\mathrm{a}} / \ln 2\right) \ln t}, \\
d r=\frac{R_{\mathrm{r}}^{2}\left(W_{\mathrm{a}} / \ln 2\right) d t}{t\left(R_{\mathrm{r}}+\left(W_{\mathrm{a}} / \ln 2\right) \ln t\right)^{2}},
\end{gathered}
$$

and integration bounds $\lim _{r \rightarrow 0} t(r)=1$ and $\lim _{r \rightarrow R_{\mathrm{r}}} t(r) \rightarrow$ $\infty, C_{\mathrm{av}}$ can be rewritten as follows:

$$
C_{\mathrm{av}}=R_{\mathrm{r}}^{2} \frac{W_{\mathrm{a}}}{\ln 2} \int_{1}^{\infty} \frac{e^{-(t-1) / \bar{\gamma}_{\mathrm{a}}} d t}{t\left(R_{\mathrm{r}}+\left(W_{\mathrm{a}} / \ln 2\right) \ln t\right)^{2}} .
$$

After integration by parts, (30) can be expressed in the form

$$
C_{\mathrm{av}}=R_{\mathrm{r}}\left(1-\frac{1}{\bar{\gamma}_{\mathrm{a}}} e^{1 / \bar{\gamma}_{\mathrm{a}}} \int_{1}^{\infty} \frac{e^{-t / \bar{\gamma}_{\mathrm{a}}} d t}{1+\left(W_{\mathrm{a}} / R_{\mathrm{r}} \ln 2\right) \ln t}\right) .
$$

Since a closed form expression for the definite integral in (31) does not exist, we have deduced a tight lower bound for the mean e2e data rate that also serves as a good analytical approximation as given in Section 4.5. Let us now consider the distribution for the relative time $\left(\tau_{\mathrm{r}}=T_{\mathrm{r}} / T\right)$, which is used to determine the allocation of time resources for the RL transmission. According to (11), we have $\tau_{\mathrm{r}}=R / R_{\mathrm{r}}$ and, thus,

$$
F_{\tau_{\mathrm{r}}}(\tau)=\frac{1}{R_{\mathrm{r}}} F_{R}\left(\frac{\tau}{R_{\mathrm{r}}}\right) .
$$

For the AL, the time sharing $\tau_{\mathrm{a}}=T_{\mathrm{a}} / T$ can be computed from $\tau_{\mathrm{a}}=1-\tau_{\mathrm{r}}$. The CDF presented in (32) can be used for dimensioning purposes in the cellular network planning phase: We may require, for example, that the relative transmission time in the RL is under some predefined threshold $\tau_{\mathrm{r}}^{\max }$ with a given probability $P_{\mathrm{r}}^{\max }$. The reason for such a requirement may be the necessity of saving some RL resources to serve those UEs that are directly connected to BS that acts as donor of the RN. We note that (32) also characterizes the relative data rate loss due to the maximum data rate that the RL is able to support. Namely, if ideal RL is replaced by a link with a finite data rate $R_{\mathrm{r}}$, the loss in the e2e data rate is $R_{\mathrm{a}}-R$, and the relative loss is $\delta R=\left(R_{\mathrm{a}}-R\right) / R_{\mathrm{a}}=R / R_{\mathrm{r}}$. The relative data rate loss can be used as a performance indicator when contrasting the data rate that a RN (with data rate limitation in the RL) and a pico-base station (without data rate limitation in the backhaul) are able to provide. 


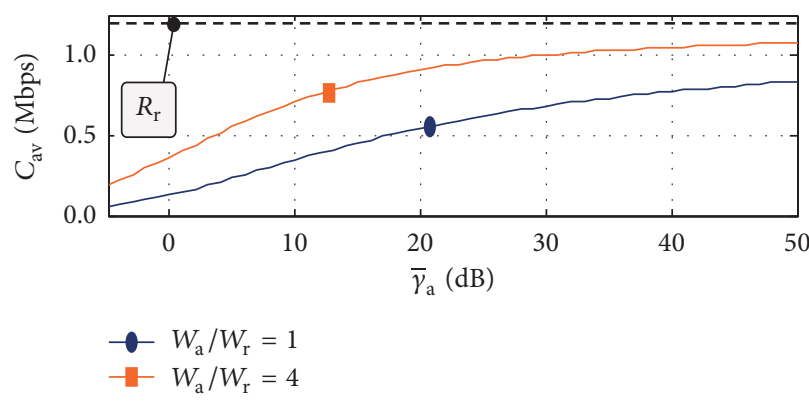

(a)

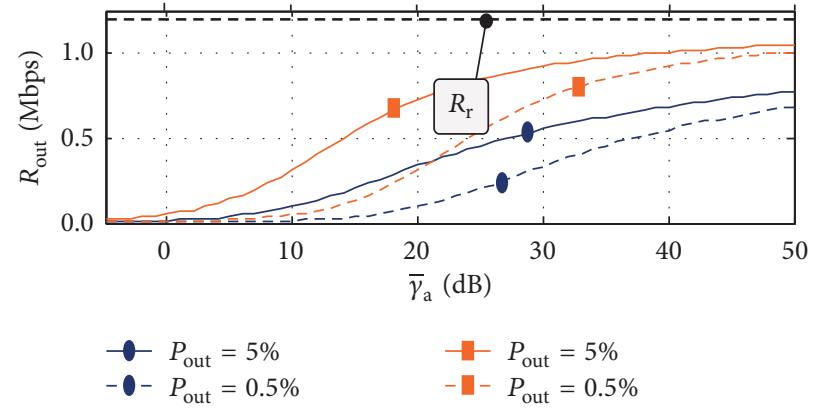

(b)

Figure 5: Mean (average) e2e data rate (a) and outage e2e data rate (b) as a function of AL mean SNR; $W_{\mathrm{r}}=180 \mathrm{kHz}$ and $\bar{\gamma}_{\mathrm{r}}=20 \mathrm{~dB}$.

4.4. Outage Data Rate for Optimal RA Scheme. The outage data rate is defined as a largest data rate $R^{\text {out }}$ such that $P(R<$ $\left.R^{\text {out }}\right)=P^{\text {out }}$ is verified, where $P^{\text {out }}$ is the outage probability [36]. In order to derive an expression for $R^{\text {out }}$, we start by writing

$$
\begin{aligned}
P^{\text {out }} & =P\left(R_{\mathrm{e} 2 \mathrm{e}}^{\text {opt }}<R^{\text {out }}\right)=P\left(\frac{R_{\mathrm{a}} \cdot R_{\mathrm{r}}}{R_{\mathrm{a}}+R_{\mathrm{r}}}<R^{\text {out }}\right) \\
& =P\left(R_{\mathrm{a}}<\frac{R_{\mathrm{r}} \cdot R^{\text {out }}}{R_{\mathrm{r}}-R^{\text {out }}}\right) \\
& =F_{\gamma_{\mathrm{a}}}\left(e^{\left(\ln 2 / W_{\mathrm{a}}\right) \cdot\left(\left(R_{\mathrm{r}} \cdot R^{\text {out }}\right) /\left(R_{\mathrm{r}}-R^{\text {out }}\right)\right)}-1\right),
\end{aligned}
$$

where the latter equality follows from (23). The outage data rate $R^{\text {out }}$ can be analytically computed from (33) provided that the inverse of $F_{\gamma_{a}}(\gamma)$ admits a closed form expression. If the SNR of AL is assumed to be exponentially distributed (Rayleigh fading), then we find that

$$
\begin{aligned}
R^{\text {out }} & =\frac{R_{\mathrm{a}}^{\text {out }} \cdot R_{\mathrm{r}}}{R_{\mathrm{a}}^{\text {out }}+R_{\mathrm{r}}}, \\
& =\frac{\left(W_{\mathrm{a}} / \ln 2\right) \ln \left(1-\bar{\gamma}_{\mathrm{a}} \ln \left(1-P^{\text {out }}\right)\right) \cdot R_{\mathrm{r}}}{\left(W_{\mathrm{a}} / \ln 2\right) \ln \left(1-\bar{\gamma}_{\mathrm{a}} \ln \left(1-P^{\text {out }}\right)\right)+R_{\mathrm{r}}},
\end{aligned}
$$

where $R_{\mathrm{a}}^{\text {out }}$ is the outage data rate obtained as a solution from

$$
\begin{aligned}
R_{\mathrm{a}}^{\text {out }} & =W_{\mathrm{a}} \cdot \log _{2}\left(1+\gamma_{\mathrm{a}}^{\text {out }}\right), \\
F_{\gamma_{\mathrm{a}}}\left(\gamma_{\mathrm{a}}^{\text {out }}\right) & =P^{\text {out }} .
\end{aligned}
$$

Thus, from (34) and (35), we see that e2e data rate formula (12) defines a direct relation between the outage data rate in the $\mathrm{AL}$ and the e2e link. Let us now come back to the discussion that we had on the time sharing between RL and AL. Similarly, we note that, by using the distribution (32), it is possible to compute with (34) the time sharing threshold $\tau_{r}^{\max }$ that should be verified for a given excess probability $P_{r}^{\max }$.

Figure 5 shows the mean (average) e2e data rate $\left(C_{\mathrm{av}}\right)$ and the outage e2e data rate $\left(R^{\text {out }}\right)$ as a function of the mean SNR that is experienced in the AL. In all cases, it is considered that the mean SNR in the RL is $20 \mathrm{~dB}$. The performance upper limits for $C_{\mathrm{av}}$ and $R^{\text {out }}$, which depend on the mean SNR of the RL, are indicated with black dashed lines. One more time, we have plotted curves for the cases $W_{\mathrm{a}} / W_{\mathrm{r}}=1$ (dark blue curves) and $W_{\mathrm{a}} / W_{\mathrm{r}}=4$ (orange curves), respectively.

Figure 5(a) shows that the mean e2e data rate with optimal RA can be clearly increased by using additional frequency resources in the AL. Let us consider two cases: $C_{\mathrm{av}}=0.25 \mathrm{Mbps}$ and $C_{\mathrm{av}}=0.75 \mathrm{Mbps}$. We note that $C_{\mathrm{av}}=$ $0.25 \mathrm{Mbps}$ is obtained when $\bar{\gamma}_{\mathrm{a}}=-1 \mathrm{~dB}$ for $W_{\mathrm{a}} / W_{\mathrm{r}}=4$ and $\bar{\gamma}_{\mathrm{a}}=7 \mathrm{~dB}$ for $W_{\mathrm{a}} / W_{\mathrm{r}}=1$. However, if we target a mean data rate of $0.75 \mathrm{Mbps}$, then a mean SNR in the AL should increase to $\bar{\gamma}_{\mathrm{a}}=12 \mathrm{~dB}$ for $W_{\mathrm{a}} / W_{\mathrm{r}}=4$ and $\bar{\gamma}_{\mathrm{a}}=40 \mathrm{~dB}$ for $W_{\mathrm{a}} / W_{\mathrm{r}}=1$.

Figure 5(b) shows the outage data rate for an outage probability of $5 \%$ (solid curves) and $0.5 \%$ (dashed curves), respectively. As expected, the outage data rate $R^{\text {out }}$ reduces when decreasing the outage probability, as this condition represents a more stringent requirement in terms of Quality of Service (QoS). Since dashed and solid curves with the same color become close to each other when the mean SNR in the AL grows, we conclude that the performance loss for guaranteeing a better e2e radio link reliability (lower outage probability) can be partly compensated by allocating more resources on the AL link. We also note the positive impact that the provisioning of a larger bandwidth in the AL has when the mean SNR of the AL is low.

4.5. Lower Bound Approximation for the Mean e2e Data Rate. In order to obtain a lower bound for $C_{\mathrm{av}}$, the integration domain in (31) is divided into three subintervals, such that

$$
I=\int_{1}^{\infty} \frac{e^{-t / \bar{\gamma}_{\mathrm{a}}} d t}{1+\left(W_{\mathrm{a}} / R_{\mathrm{r}} \ln 2\right) \ln t}=I_{1}+I_{2}+I_{3}
$$

is verified. The rationale behind this consists in defining integration bounds, such that each definite integral $I_{x}$ with $x=\{1,2,3\}$ admits a closed form solution that represents an upper bound of $I$ in its corresponding integration interval. Exact expressions for $I_{1}, I_{2}$, and $I_{3}$, which are derived in the Appendix, are presented in (37), (38), and (39), respectively. 


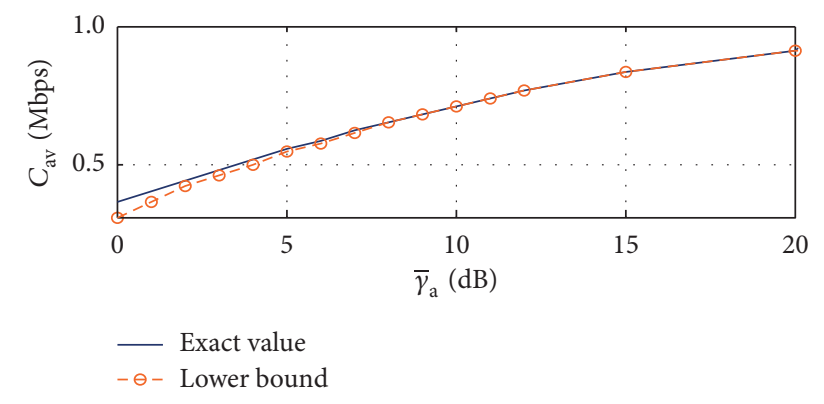

FIgURE 6: Exact mean e2e data rate and lower bound approximation given by (40). Optimization for $\bar{\gamma}_{\mathrm{a}} \in[0,20] \mathrm{dB}: m=0.995$ and $M \in$ $[2.42,2.61]$.

The first subinterval is given by $1 \leq t<m \bar{\gamma}_{\mathrm{a}}$, with $m<1$. Thus, it is possible to show that

$$
I_{1}<\sum_{n=0}^{N} \frac{(-1)^{n}}{n !}\left(\frac{1}{\bar{\gamma}_{\mathrm{a}}}\right)^{n} \int_{1}^{m \bar{\gamma}_{\mathrm{a}}} \frac{t^{n} d t}{1+\left(W_{\mathrm{a}} / R_{\mathrm{r}} \ln 2\right) \ln t},
$$

where the exponential term in (31) has been replaced by its Taylor series expansion when argument $t / \bar{\gamma}_{\mathrm{a}}<m<1$. Note that (37) provides an upper bound only when the number of terms in the sum $(N)$ is odd. The right-hand side function in (37) can be integrated analytically, and its closed form solution is given in terms of the exponential integral function $E_{i}(t)$; see (A.3) in the Appendix. The second subinterval is $m \bar{\gamma}_{\mathrm{a}} \leq t<M \bar{\gamma}_{\mathrm{a}}$, with $M>1$. Thus,

$$
I_{2}<\int_{m \bar{\gamma}_{\mathrm{a}}}^{M \bar{\gamma}_{\mathrm{a}}} \frac{e^{-t / \bar{\gamma}_{\mathrm{a}}} d t}{1+\left(W_{\mathrm{a}} / R_{\mathrm{r}} \ln 2\right) \Psi(t)},
$$

where $\Psi(t)$ represents a linear (first-order) interpolation for the logarithmic function, such that $\Psi(t)=\ln \left(m \bar{\gamma}_{\mathrm{a}}\right)$ and $\Psi\left(M \bar{\gamma}_{\mathrm{a}}\right)=\ln \left(M \bar{\gamma}_{\mathrm{a}}\right)$ are verified. Since the logarithmic function is concave, $\Psi(t)<\ln t$ on $\left(m \bar{\gamma}_{\mathrm{a}}, M \bar{\gamma}_{\mathrm{a}}\right)$ and, hence, (38) is valid. Its solution is given in Appendix; see (A.8). The third subinterval is given by $M \bar{\gamma}_{\mathrm{a}} \leq t<\infty$. Thus,

$$
\begin{aligned}
I_{3} & <\int_{M \bar{\gamma}_{\mathrm{a}}}^{\infty} \frac{e^{-t / \bar{\gamma}_{\mathrm{a}}} d t}{1+\left(W_{\mathrm{a}} / R_{\mathrm{r}} \ln 2\right) \ln \left(M \bar{\gamma}_{\mathrm{a}}\right)} \\
& =\frac{\bar{\gamma}_{\mathrm{a}} e^{-M}}{1+\left(W_{\mathrm{a}} / R_{\mathrm{r}} \ln 2\right) \ln \left(M \bar{\gamma}_{\mathrm{a}}\right)} .
\end{aligned}
$$

After combining (37)-(39), the lower bound expression for $C_{\mathrm{av}}$ is obtained as follows:

$$
C_{\mathrm{av}}>C_{\mathrm{LB}}=R_{\mathrm{r}}\left[1-\frac{1}{\bar{\gamma}_{\mathrm{a}}} e^{1 / \bar{\gamma}_{\mathrm{a}}}\left(I_{1}+I_{2}+I_{3}\right)\right] .
$$

Closed form expressions for the definite integrals in (37) and (38) have been derived in the Appendix; see (A.3) and (A.8). Figure 6 shows that the accuracy of $C_{\mathrm{LB}}$ increases rapidly with $\bar{\gamma}_{\mathrm{a}}$, with a relative error that is lower than $2 \%$ for $\bar{\gamma}_{\mathrm{a}}>2 \mathrm{~dB}$ (practical mean SNR values for the AL). Optimal values of $m$ and $M$ have been found using numerical methods.

\section{Conclusion}

In this paper, a comparative analysis of several RA schemes (i.e., conventional RA, fixed RA with and without buffer, and optimal RA) was presented in the context of a two-hop DF infrastructure relaying system. Based on the obtained results, it is possible to conclude that the optimal RA scheme that was presented provides a notable performance gain when compared to the other baseline RA schemes, which kept the communication resources in both RL and AL fixed or adjusted them slowly in long-term. Moreover, it was possible to derive closed form expressions for the CDF and PDF of the e2e data rate when optimal RA scheme is used. This work also contributes by calculating the mean and outage data rate for the e2e relaying link.

In infrastructure relaying systems, like the ones considered in $4 \mathrm{G}$ networks, the RL easily becomes the performance bottleneck; this is because the macrocellular BS allocates less frequency resources in the RL, when compared to the resources that the $\mathrm{RN}$ can allocate in the AL. This follows from the fact that a macrocellular BS must share its frequency resources with many RNs and UEs simultaneously, whereas a RN usually serves a smaller geographical area and has the possibility of allocating more bandwidth per UE. Accordingly, we found that the performance impact of the available bandwidths in both RL and AL was notable. With the aid of the optimal RA scheme, it was possible to show that the efficiency of RL usage can be notably improved. This effect has shown a very positive impact on the e2e data rate performance of the relaying system.

Finally, a tight lower bound approximation for the mean e2e data rate of the two-hop DF relaying system was obtained using a novel theoretical approach. These results shed light on the complex relationship among design parameters and system variables, thus providing key insights into infrastructure relaying dimensioning and the role of Radio Resource Management (RRM) within contemporary heterogeneous networks.

\section{Future Work in RA: Some 5G Relaying Challenges}

Although the study in this paper is generic in nature and is based on mathematical analysis, our work is largely motivated by the $4 \mathrm{G}$ LTE infrastructure relaying system model. That is, it is considered that half-duplex DF RNs are placed in large macrocells, where the so-called Donor eNode B serves the RN over the RL, and the RN itself acts as any other small eNode B for the perspective of the UE. In 5G, some newly introduced challenges will likely take place. From the relaying point of view, it is expected that main challenges will be related to the $5 \mathrm{G}$ features, such as communication using higher frequency radio carrier (mmWave), tactile Internet, mobile RNs, and relaying in Device-to-Device (D2D) communication.

In mmWave communication, high-frequency carriers are preferably used in small cells, where extremely high data rates are expected to be transported using large bandwidths. This poses challenges on the RA scheme that should be used over 
the RL and AL. Let us give an example: If, for instance, a small $\mathrm{RN}$ is placed on a lamp post, then UEs demanding very high data rates may appear and disappear very fast. Since in this scenario it is very likely to have few RNs in range (located on nearby lamp posts), the incoming high data rate per UE can be predicted. That is, the system may apply predictive RA over several RNs. Yet, design of such system will be very challenging. We also note that the frequency spectrum usage in the RL and AL may be more complex in $5 \mathrm{G}$ than in $4 \mathrm{G}$. For example, the RL and AL may be operated on different radio carriers (so-called outband relaying), to fit the RL and $\mathrm{AL}$ data rates and/or to share the load in different frequency carriers.

Tactile Internet refers to the combination of extremely low latency, high availability, good reliability, and strong security in the provision of Internet services. From the perspective of a relaying system, the main challenges are coming from the low-latency and reliability requirements. By nature, the delays of the radio protocol (in, e.g., RA) are larger in two-hop transmission rather than in direct transmission. Even, more importantly, due to strict latency requirements, the effective use of buffers to store data temporarily in a $\mathrm{RN}$ is challenging as it may increase the communication latency notably. Finally, to keep the two-hop communication reliable, the RL and AL, as well as the RA that is utilized, should be designed carefully.

RNs in mobile relaying are not static by definition. Then, the data rate that can be supported in the RL may change in time and, as a consequence, the implementation of the optimal RA scheme over RL and AL becomes a challenge. Moreover, network protocols like the ones used in the handover process pose further challenges on the RA for the RL. Relaying in D2D creates totally new RA problems: we may need to identify the best RN (among many candidates), take into account the source node, $\mathrm{RN}$, and destination node that may be moving, and change the active source node dynamically when its feasibility to serve the RN decreases for some reason. Device relaying may also have limited buffering capabilities.

\section{Appendix}

The right-hand side function in (37) can be integrated to obtain a closed form for $I_{1}$ by setting $b=W_{\mathrm{a}} / R_{\mathrm{r}} \ln 2$ as

$$
I_{1}<K\left(\int_{0}^{m \bar{\gamma}_{\mathrm{a}}} \frac{t^{n} d t}{1+b \ln t} d t-\int_{0}^{1} \frac{t^{n} d t}{1+b \ln t} d t\right)
$$

where $K=\sum_{n=0}^{N}\left((-1)^{n} / n !\right)\left(1 / \bar{\gamma}_{\mathrm{a}}\right)^{n}$. Then, by substituting $s=$ $\left(t / m \overline{\gamma_{\mathrm{a}}}\right)$ and $d t=m \overline{\gamma_{\mathrm{a}}} d s$, it is possible to see that

$$
\begin{aligned}
I_{1} & <K\left(\int_{0}^{1} \frac{\left(m \overline{\gamma_{\mathrm{a}}} s\right)^{n}}{1+b \ln \left(m \overline{\gamma_{\mathrm{a}}} s\right)} m \overline{\gamma_{\mathrm{a}}} d s\right. \\
& \left.-\int_{0}^{1} \frac{t^{n} d t}{1+b \ln t} d t\right)
\end{aligned}
$$

$$
\begin{aligned}
& =K\left(\frac{\left(m \overline{\gamma_{\mathrm{a}}}\right)^{n+1}}{b} \int_{0}^{1} \frac{s^{n}}{\left((1 / b)+\ln m \overline{\gamma_{\mathrm{a}}}\right)+\ln s} d s\right. \\
& \left.-\int_{0}^{1} \frac{t^{n} d t}{1+b \ln t} d t\right)
\end{aligned}
$$

holds. After some manipulations, in which [34, Prop. 4.281.3] is used, $I_{1}$ is upper bounded as

$$
\begin{aligned}
I_{1} & <\sum_{n=0}^{N} \frac{(-1)^{n}}{n !}\left(\frac{1}{\bar{\gamma}_{\mathrm{a}}}\right)^{n} \\
& \cdot \frac{1}{b} e^{-n / b}\left(E_{i}\left(n \ln \left(e^{1 / b} m \overline{\gamma_{\mathrm{a}}}\right)\right)-E_{i}\left(\frac{n}{b}\right)\right) .
\end{aligned}
$$

The term $I_{2}$ has been calculated using a linear interpolation $\Psi(t)$ for the logarithmic function on interval $\left(t_{1}=m \overline{\gamma_{\mathrm{a}}}, t_{2}=\right.$ $\left.M \overline{\gamma_{\mathrm{a}}}\right)$, such that $\Psi\left(m \overline{\gamma_{\mathrm{a}}}\right)=\ln \left(m \overline{\gamma_{\mathrm{a}}}\right)$, and hence

$$
I_{2}<\int_{m \overline{\gamma_{\mathrm{a}}}}^{M \overline{\gamma_{\mathrm{a}}}} \frac{e^{-t / \overline{\gamma_{\mathrm{a}}}} d t}{1+b \Psi(t)}=\int_{m \overline{\gamma_{\mathrm{a}}}}^{M \overline{\gamma_{\mathrm{a}}}} \frac{e^{-t / \overline{\gamma_{\mathrm{a}}}} d t}{1+b(p t+q)} .
$$

Under the assumption of linear interpolation that is proposed, it is possible to show that

$$
\begin{aligned}
& p=\frac{\ln (M / m)}{(M-m) \overline{\gamma_{\mathrm{a}}}}, \\
& q=\ln \left(m \overline{\gamma_{\mathrm{a}}}\right)-\left(\frac{m}{M-m}\right) \ln \left(\frac{M}{m}\right) .
\end{aligned}
$$

Combining the expressions for $p, q$ and the approximation of the exponential integral function [34]

$$
\int \frac{e^{C Z}}{A Z+B} d Z=\frac{1}{A} E_{i}\left(C\left(\frac{B}{A}+Z\right)\right) e^{-B C / A},
$$

as well as further mathematical manipulations, the upper bound approximation for the definite integral presented in (A.4) can be rewritten as follows:

$$
\begin{aligned}
& I_{2} \\
& <\int_{m \overline{\gamma_{\mathrm{a}}}}\left(\frac{e^{-t / \overline{\gamma_{\mathrm{a}}}} d t}{1+b\left(\left(k_{1} t / k_{2} \overline{\gamma_{\mathrm{a}}}\right)+\ln \left(m \overline{\gamma_{\mathrm{a}}}\right)-\left(k_{1} m / k_{2}\right)\right)}\right),
\end{aligned}
$$

where $k_{1}=\ln (M / m)$ and $k_{2}=M-m$. Thus,

$$
\begin{gathered}
I_{2}<\left(\frac{k_{2} \overline{\gamma_{\mathrm{a}}}}{b k_{1}}\right) e^{\left(\left(k_{2}+b M \ln \left(m \overline{\gamma_{\mathrm{a}}}\right)-b m \ln \left(M \overline{\overline{\mathrm{a}}_{\mathrm{a}}}\right)\right) / b k_{1}\right)}\left(E _ { 1 } \left(k_{2}\right.\right. \\
\left.\left.\cdot \frac{1+b \ln \left(m \overline{\gamma_{\mathrm{a}}}\right)}{b k_{1}}\right)-E_{1}\left(k_{2} \frac{1+b \ln \left(M \overline{\gamma_{\mathrm{a}}}\right)}{b k_{1}}\right)\right)
\end{gathered}
$$

is finally obtained.

\section{Conflicts of Interest}

The authors declare that they have no conflicts of interest. 


\section{Acknowledgments}

This work was supported by the Academy of Finland under Grants 287249 and 311752.

\section{References}

[1] Ericsson mobility report, 2017, https://www.ericsson.com/ assets/local/mobility-report/documents/2017/ericsson-mobilityreport-june-2017.pdf.

[2] "Cisco visual networking index: global mobile data traffic forecast update, 2016-2021," White Paper, 2017.

[3] J. M. C. Brito, "Trends in wireless communications towards 5G networks-The influence of e-health and IoT applications," in Proceedings of the 1st International Multidisciplinary Conference on Computer and Energy Science, SpliTech 2016, July 2016.

[4] J. Sydir, IEEE 802.16 Broadband Wireless Access Working Group: Harmonized contribution on 802.16j (Mobile Multihop Relay) usage models," September, 2006.

[5] A. B. Saleh, S. Redana, J. Hämäläinen, and B. Raaf, "On the coverage extension and capacity enhancement of inband relay deployments in LTE-advanced networks," Journal of Electrical and Computer Engineering, vol. 2010, Article ID 894846, 12 pages, 2010.

[6] Z. Hasan, H. Boostanimehr, and V. K. Bhargava, "Green cellular networks: a survey, some research issues and challenges," IEEE Communications Surveys \& Tutorials, vol. 13, no. 4, pp. 524-540, 2011.

[7] S. McLaughlin, P. M. Grant, J. S. Thompson et al., "Techniques for improving cellular radio base station energy efficiency," IEEE Wireless Communications Magazine, vol. 18, no. 5, pp. 10-17, 2011.

[8] A. Bou Saleh, Ö. Bulakci, J. Hämäläinen, S. Redana, and B. Raaf, "Analysis of the impact of site planning on the performance of relay deployments," IEEE Transactions on Vehicular Technology, vol. 61, no. 7, pp. 3139-3150, 2012.

[9] M. Liu, J. Zhang, and P. Zhang, "Outage probability of dual-hop multiple antenna relay systems with interference at the relay and destination," International Journal of Antennas and Propagation, vol. 2014, Article ID 370684, 7 pages, 2014.

[10] Q. Cui, X. Yang, J. Hämäläinen, X. Tao, and P. Zhang, "Optimal energy-efficient relay deployment for the bidirectional relay transmission schemes," IEEE Transactions on Vehicular Technology, vol. 63, no. 6, pp. 2625-2641, 2014.

[11] Ö. Bulakci, A. B. Saleh, S. Redana, B. Raaf, and J. Hämäläinen, "Resource sharing in LTE-Advanced relay networks: Uplink system performance analysis," Transactions on Emerging Telecommunications Technologies, vol. 24, no. 1, pp. 32-48, 2013.

[12] D. Qin and Y. Wang, "Capacity Analysis of Two-Hop Multichannel Relaying with Subchannel Pairing," IEEE Communications Letters, vol. 19, no. 10, pp. 1846-1849, 2015.

[13] S. Wang, H. Peng, and X. Liu, "Sum-power minimization in multiuser single-DF-relay networks with direct links," International Journal of Distributed Sensor Networks, vol. 2016, Article ID 8467294, 7 pages, 2016.

[14] M. R. Bhatnagar, R. K. Mallik, and O. Tirkkonen, "Performance evaluation of best-path selection in a multihop decode-andforward cooperative system," IEEE Transactions on Vehicular Technology, vol. 65, no. 4, pp. 2722-2728, 2016.

[15] M. R. Bhatnagar, "Performance analysis of a path selection scheme in multi-hop decode-and-forward protocol," IEEE Communications Letters, vol. 16, no. 12, pp. 1980-1983, 2012.
[16] B. R. Manoj, R. K. Mallik, and M. R. Bhatnagar, "Buffer-aided multi-hop DF cooperative networks: a state-clustering based approach," IEEE Transactions on Communications, vol. 64, no. 12, pp. 4997-5010, 2016.

[17] X. Liu, "Outage behavior of LTE-A with non-identical rician relay links," in Proceedings of the 83rd IEEE Vehicular Technology Conference, VTC Spring 2016, pp. 1-5, May 2016.

[18] M. R. Bhatnagar, "On the capacity of decode-and-forward relaying over Rician fading channels," IEEE Communications Letters, vol. 17, no. 6, pp. 1100-1103, 2013.

[19] Y. Zhang, F. Ke, S. Feng, and X. Zhu, "Tight bounds for ergodic capacity of cooperative relaying over rayleigh fading channels," in Proceedings of the 2014 6th International Conference on Computational Intelligence and Communication Networks, CICN 2014, pp. 313-316, November 2014.

[20] J. Yang, Y. Wang, and Z. Xie, "Performance analysis of dual-hop $\mathrm{df}$ relaying in $\mathrm{g}$ fading channel in the presence of co-channel interference," in Proceedings of the International Conference on Wireless Communications \& Signal Processing (WCSP), pp. 1-6, December 2013.

[21] Y. Sun, X. Zhong, X. Chen, S. Zhou, and J. Wang, "Ergodic capacity of decode-and-forward relay strategies over general fast fading channels," IEEE Electronics Letters, vol. 47, no. 2, pp. 148-150, 2011.

[22] G. Farhadi and N. C. Beaulieu, "On the ergodic capacity of multi-hop wireless relaying systems," IEEE Transactions on Wireless Communications, vol. 8, no. 5, pp. 2286-2291, 2009.

[23] N. Yi, Y. Ma, and R. Tafazolli, "Joint rate adaptation and bestrelay selection using limited feedback," IEEE Transactions on Wireless Communications, vol. 12, no. 6, pp. 2797-2805, 2013.

[24] Y. Ma, R. Tafazolli, Y. Zhang, and C. Qian, "Adaptive modulation for opportunistic decode-and-forward relaying," IEEE Transactions on Wireless Communications, vol. 10, no. 7, pp. 2017-2022, 2011.

[25] T. Beniero, S. Redana, J. Hämäläinen, and B. Raaf, "Effect of relaying on coverage in 3GPP LTE-advanced," in Proceedings of the VTC Spring 2009 - IEEE 69th Vehicular Technology Conference, pp. 1-5, April 2009.

[26] 3GPP, "Further advancements for E-UTRA physical layer aspects (Rel. 9)," TSG RAN, December 2016.

[27] L. J. Greenstein, S. S. Ghassemzadeh, V. Erceg, and D. G. Michelson, "Ricean $\mathrm{K}$-factors in narrow-band fixed wireless channels: theory, experiments, and statistical models," IEEE Transactions on Vehicular Technology, vol. 58, no. 8, pp. 40004012, 2009.

[28] M. K. Simon and M.-S. Alouini, Digital Communication over Fading Channels, vol. 95, John Wiley \& Sons, 2005.

[29] A. F. Molisch, Wireless Communications, John Wiley \& Sons, 2007.

[30] A. Osseiran, J. F. Monserrat, and Y. P. Marsch, 5G Mobile and Wireless Communications Technology, Cambridge University Press, Cambridge, UK, 2016.

[31] N. Zlatanov, R. Schober, and P. Popovski, "Buffer-aided relaying with adaptive link selection," IEEE Journal on Selected Areas in Communications, vol. 31, no. 8, pp. 1530-1542, 2013.

[32] N. Nomikos, T. Charalambous, I. Krikidis et al., "A survey on buffer-aided relay selection," IEEE Communications Surveys \& Tutorials, vol. 18, no. 2, pp. 1073-1097, 2015.

[33] K. Loa, C. C. Wu, and S. T. Sheu, "IMT-advanced relay standards [WiMAX/LTE update]," IEEE Communications Magazine, vol. 48, no. 8, pp. 40-48, 2010. 
[34] I. S. Gradshtein and I. M. Ryzhik, Tables of Integrals, Sums, Series, and Products, 7th edition, 2007.

[35] A. Papoulis and S. Pillai, Probability, Random Variables, and Stochastic Processes, McGraw-Hill, New York, NY, USA, 4th edition, 2002.

[36] D. Tse and P. Viswanath, Fundamentals of Wireless Communication, Cambridge University Press, Cambridge, UK, 2005. 

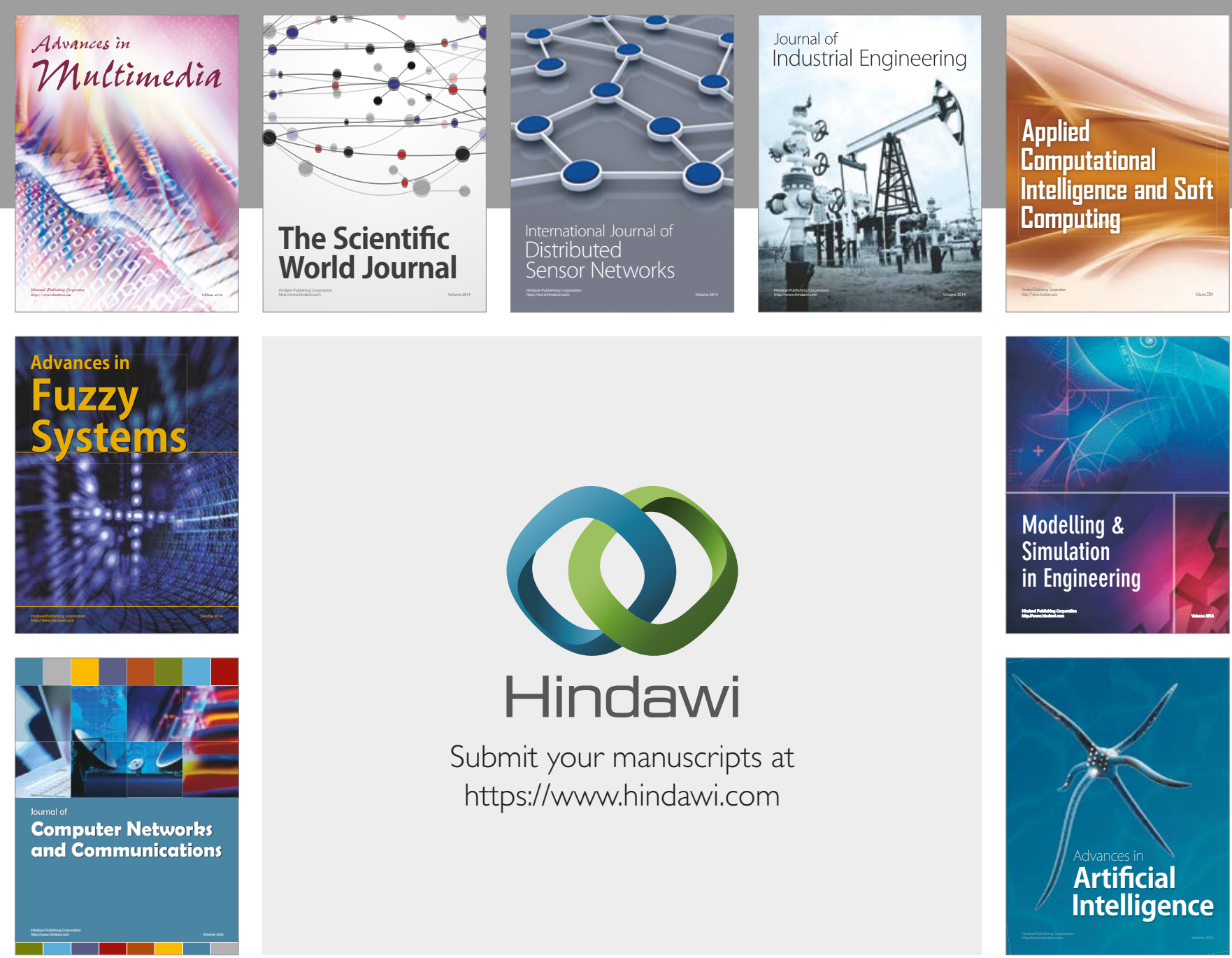

\section{Hindawi}

Submit your manuscripts at

https://www.hindawi.com
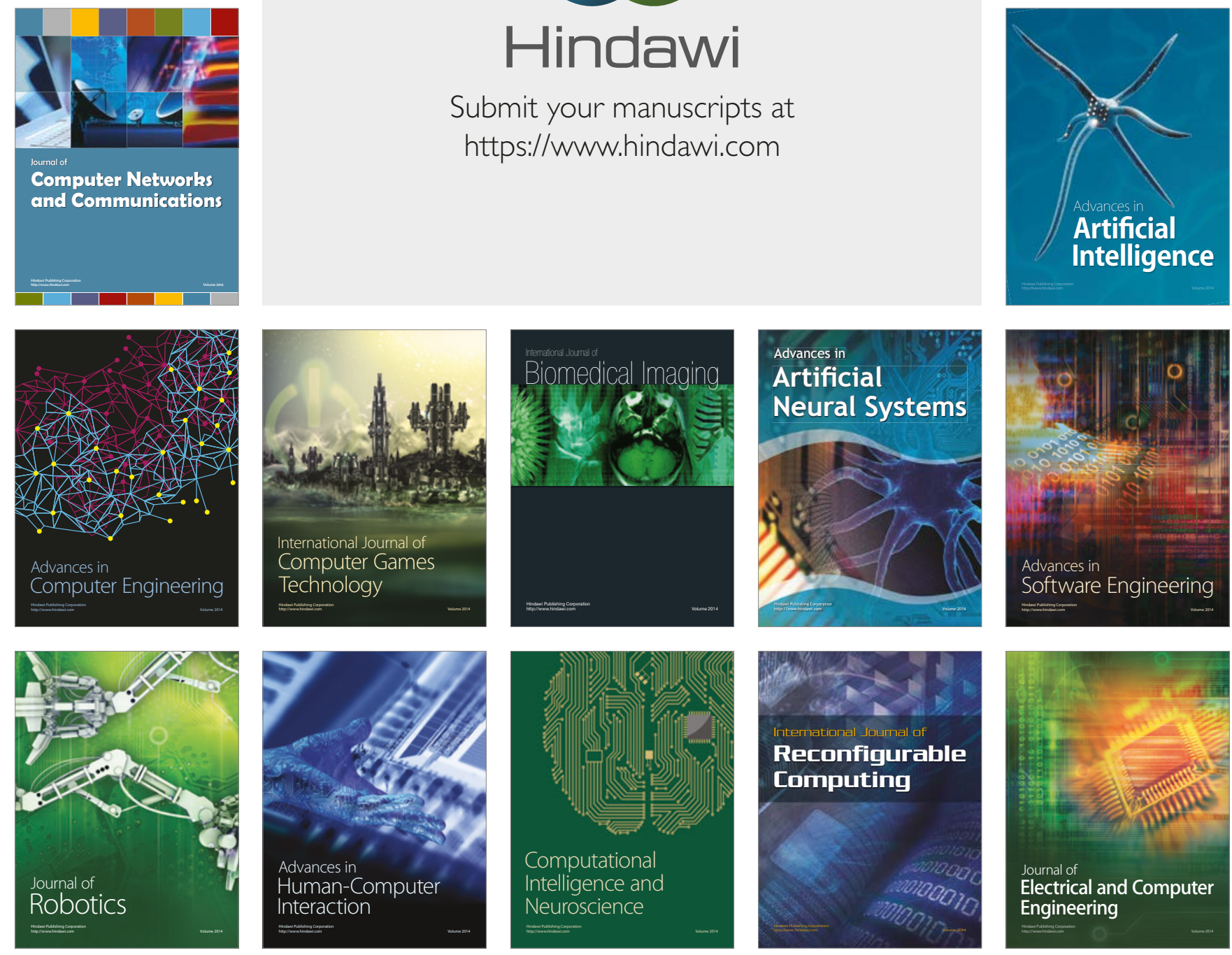